\title{
MULTI-SCALE MODELS AND SIMULATIONS OF NUCLEAR FUELS
}

\author{
MARIUS STAN \\ Los Alamos National Laboratory \\ Los Alamos, NM 87545, United States of America \\ E-mail : mastan@lanl.gov
}

Received January 22, 2009

\begin{abstract}
Theory-based models and high performance simulations are briefly reviewed starting with atomistic methods, such as Electronic Structure calculations, Molecular Dynamics, and Monte Carlo, continuing with meso-scale methods, such as Dislocation Dynamics and Phase Field, and ending with continuum methods that include Finite Element and Finite Volume. Special attention is paid to relating thermo-mechanical and chemical properties of the fuel to reactor parameters. By inserting atomistic models of point defects into continuum thermo-chemical calculations, a model of oxygen diffusivity in $\mathrm{UO}_{2+\mathrm{x}}$ is developed and used to predict point defect concentrations, oxygen diffusivity, and fuel stoichiometry at various temperatures and oxygen pressures. The simulations of coupled heat transfer and species diffusion demonstrate that including the dependence of thermal conductivity and density on composition can lead to changes in the calculated centerline temperature and thermal expansion displacements that exceed 5\%. A review of advanced nuclear fuel performance codes reveals that the many codes are too dedicated to specific fuel forms and make excessive use of empirical correlations in describing properties of materials. The paper ends with a review of international collaborations and a list of lessons learned that includes the importance of education in creating a large pool of experts to cover all necessary theoretical, experimental, and computational tasks.
\end{abstract}

KEYWORDS : Nuclear Fuels, Models, Simulations, Fuel Performance, International Collaborations

\section{INTRODUCTION}

In nuclear reactors, severe radiation environments continuously alter thermo-mechanical and chemical properties of nuclear fuel materials (actinide based alloys and ceramics) [1]. The physics and chemistry of such materials increase in complexity due to irradiation effects. To address these issues, several projects have been developed all over the world to assess the properties of multi-component actinide based ceramics. However, most assessments still involve fitting of data followed by extrapolations or interpolations into new temperature, composition or pressure regimes.

One of the most challenging aspects of developing a comprehensive understanding of nuclear fuels is their complex, evolving composition. The study of multicomponent systems containing $\mathrm{U}, \mathrm{Np}, \mathrm{Pu}, \mathrm{Am}, \mathrm{Cm}$ and their oxides, nitrides, and alloys, is considerably complicated by the presence of fission products such as $\mathrm{Xe}, \mathrm{Cs}, \mathrm{Sr}, \mathrm{He}, \mathrm{I}$, and Tc. Most commercial reactor and fuel performance codes assume the fuel to be a homogenous material and average the properties over the computational domain. By including the heterogeneous character of the fuels, the precision and accuracy of predictions can be significantly improved. This is even more important for simulations of dispersed, inert matrix, and micro(nano)structured fuels.

Most of the theoretical and computational results published over the last few decades refer to actinide based oxides; much less attention was paid to nitrides and carbides, and very little to metallic fuels. That is a reflection of the overwhelming presence of oxide fuels in existing nuclear reactors. However, part of the new generation of fast nuclear reactors, as well as the reactors for space propulsion, require non-oxide fuels.

In this work we submit that a multi-physics approach to developing a fundamental understanding of properties of complex nuclear fuel materials in the reactor environment, will lead to improved tools for predicting phenomena such as heat transfer, phase stability, species diffusion, and fission products retention.

Recently, combined theoretical, computational, and experimental efforts have provided valuable information about materials properties and important phenomena associated with nuclear fuels. However, as of today, models and simulations are not regarded as critical tools for fuel design and optimization. One of the reasons is the lack of predictability, often associated with the empirical correlations used in computational the models. The applicability of such correlations is limited to a regime 
where experimental data is available. Past attempts to extrapolate outside this regime have sometimes led to faulty predictions and costly engineering decisions.

The idea of replacing empirical correlations with theory-based models for the purpose of improving high performance simulation tools is not new. For example, in USA, the ban on nuclear weapons experiments led to the "stockpile stewardship" policy and the Advanced Scientific Computing (ASC) program. For various reasons, ASC did not have a counterpart in the nuclear fuels area. No doubt that, building on the ASC experience, advanced nuclear fuel performance codes can incorporate theorybased models and run simulations on high performance computational platforms.

\section{MODELS AND SIMULATIONS}

The definitions below are intended to ensure a coherent framework for the presentation of results and discussions in this paper:

A model is a logical description of how a system (nuclear fuel material, in our case) performs. Models are based either on a theory or on empirical knowledge and are validated by experiment (rarely by computation). Most models are presented as mathematical expressions. For example, "enthalpy depends linearly on temperature" and "enthalpy is quadratic in temperature" are models.

Empirical models are collections of experimental observations fitted to mathematical expressions, such as (but not only) polynomial functions. When accurate, they allow for concise descriptions of materials properties and are extremely valuable for technological applications. However, empirical models are only valid within the range of parameters and irradiation conditions covered in the data-set on which they have been developed. Interpolation and extrapolation of empirical models is a dangerous practice that can lead to errors. Since the uncertainty associated with empirical models is often large, confidence intervals are difficult to calculate. Theory-based models are developed to include and explain the physics, chemistry, and materials science of fuel materials. These models are often referred to as "mechanistic" descriptions of properties. They are expected to provide a deeper understanding of the nature of the properties and to have improved predictive character.

A Simulation is the process of running computer programs to reproduce, in a simplified way, the behavior of a system. Simulations describe the evolution of the system along a certain coordinate, most often the time. For example, a simulation of heat transport in a fuel element can describe the enthalpy content of a specific volume at different moments in time. The simulation is likely to involve a model of enthalpy, similar or more sophisticated than the one proposed above.

\subsection{Theoretical and Computational Methods}

Models and simulations must address a wide range of space and time scales, starting with the nucleus, the electronic structure, atomistic and nano-scale, mesoscale, all the way to the fuel element size (centimeters), and from pico-seconds, all the way to the operating and storage characteristic times (months, years). The characteristic time step and space length associated with various phenomena cannot be strictly defined. For example, diffusion involves the electronic structure properties of the atoms at a microscopic level and nanosecond times. Still, the kinetics of bulk diffusion processes can be characterized from a continuum, macroscopic point of view using characteristic times of minutes, hours, and even days for the fuel-clad interaction.

To address all relevant properties and phenomena that occur in materials in general and nuclear fuels in particular, numerous theoretical and computational methods have been developed. The methods cover various time and space scales, as shown in Fig. 1. The information is transferred between scales via characteristic parameters such as density, energy, or grain size.

Nuclear methods are often reviewed as part of reactor physics books [2] and revolve around nuclear reactions and neutron transport theory. Sophisticated mathematical methods and computational techniques are used to calculate nuclear cross sections. Although very important for criticality calculations and reactor energy balance, the nuclear methods have, as of now, little impact on materials models. Since the thermo-mechanical and chemical properties of the nuclear fuels appear to be more influenced by the electronic structure rather than the nuclear structure of the atoms, the nuclear data and associated models will not be reviewed in this work.

Atomistic methods account for the evolution of each atom in the computation domain and typically focus on local (point) properties that are then used as a basis for statistical mechanics evaluations of bulk properties.

Electronic structure (ES) calculations are Quantum Mechanical (QM) calculations aimed at describing properties such as energy levels or bands, cohesive energy, lattice parameters, and phonon spectra. They provide invaluable information about materials, especially when there is no experimental data [3-5]. The QM information feeds into higher scale models and is often used to determine inter-atomic potentials and forces. One of the most popular approximations is the Density Functional Theory (DFT) [6,7], sometimes coupled with the direct force method $[8,9]$.

Most reliable results from electronic structure calculations are obtained at $0 \mathrm{~K}$ temperatures, for single element substances. Unfortunately, it is quite common to compare these results with room or even higher temperature experiments, in an attempt to achieve a weak form of validation. Another challenge is calculating properties of mixtures and solutions, for specific compositions. That is 
related to the small number of atoms involved in the calculation cell (often less than 100). In spite of the limitations, ES methods are the most theoretically sound and most promising tools for predicting materials properties.

Molecular Dynamics (MD) methods typically operate at time scales no larger than hundreds of picoseconds and involve up to one million atoms [10]. Although MD methods are capable of capturing lower-scale properties, they do not directly incorporate quantum mechanical properties and only operate within the framework of classical mechanics. MD methods are very powerful and can evaluate both equilibrium properties and parameters of chemical kinetics models [11].

The most challenging component of MD is defining the inter-atomic potentials and the associated inter-atomic forces. The atoms are given initial velocities and then the structure is relaxed until minimum free energy is reached. Many-body potentials, such as the Embedded Atom Method (EAM) [12] and the Modified Embedded Atom Method (MEAM) [13] have been successfully used for calculations of metals and alloys properties, but rarely for actinide based ceramics. The main reason is the very difficult process of adding charge transfer. As of today, the most reliable models for actinide based ceramics involve pair-potentials, such as the Buckingham $[14,15]$ potential.

MD methods are successful in calculating equilibrium energy and thermal properties of nuclear materials and are very efficient in studies of point defect formation and interactions. However, the MD simulations do not cover real times long enough to address phenomena such as cascade effects during irradiation or species diffusion (miliseconds or seconds). To allow for longer simulation times the Temperature Accelerated Dynamics (TAD) method increases the rate of events by increasing the temperature of the simulation [16]. The behavior at temperature of interest is then determined by a mapping technique. To allow for the investigation of more atoms, MD and TMD are often coupled with Monte Carlo (MC) [17] and Kinetic Monte Carlo (KMC) [18] methods. During MC calculations, the solution space is sampled and only points that satisfy certain criteria are accepted. The Quantum Monte Carlo method (QMC) is deemed to be the most advanced tool for evaluating properties of materials at scales that allow for direct experimental validation [19].

Meso-scale methods operate in time and space intervals that are characteristic to material's nano or micro-structure. The method are sometimes "atomistically informed", in the sense that some of the parameters in the meso-scale method are optimized against the output of atomistic calculations.

The Phase Field (PF) method is derived from the Landau theory of phase transitions and the Ginzburg-

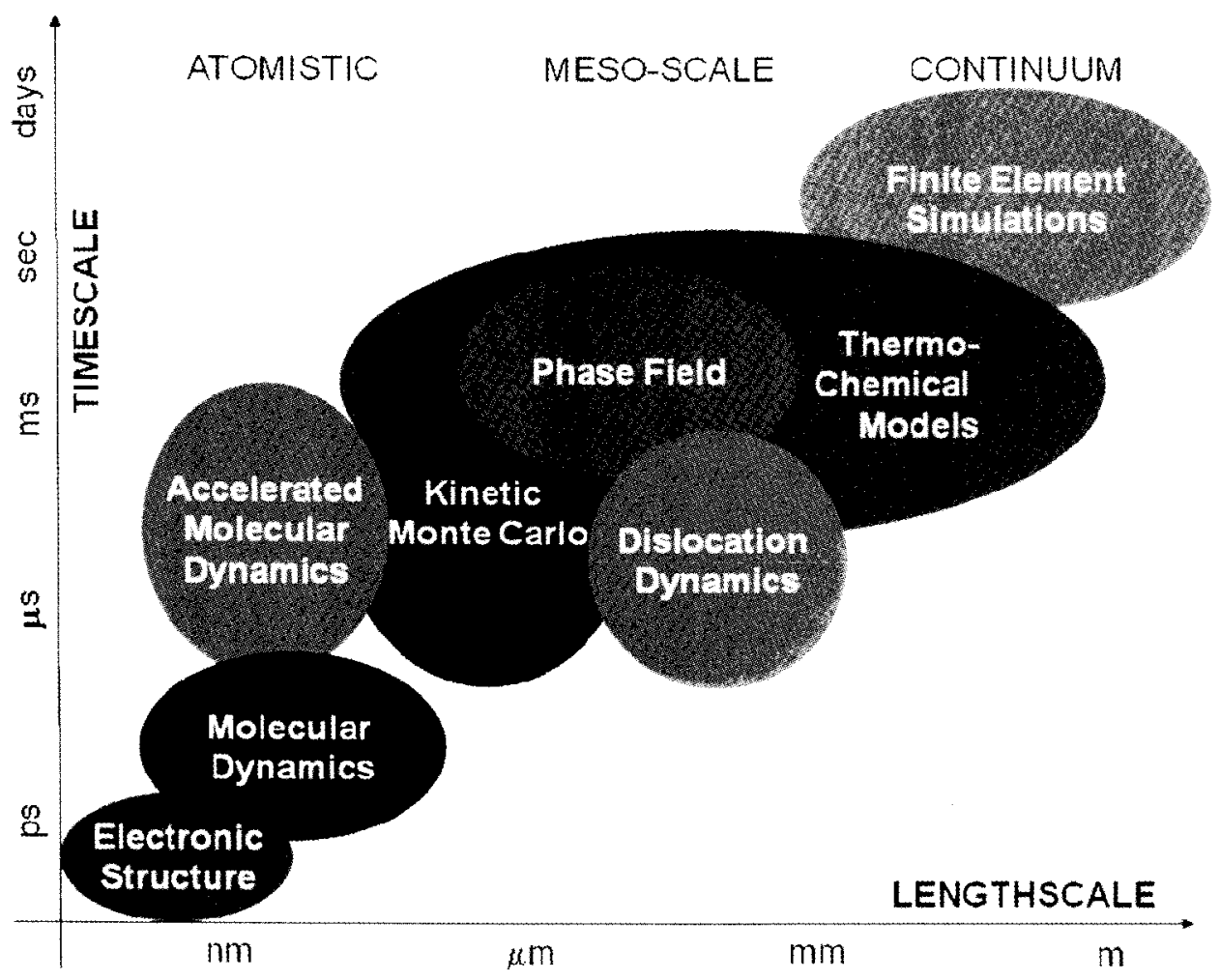

Fig. 1. Length and Time Scales Involved in Simulating Phenomena Relevant for Nuclear Materials as Covered by Computational Methods 
Landau application to superconductivity [20]. PF method assigns a set of phase variables to a target function, such as the free energy of the system, and then solves a the evolution equations. For the case of microstructure evolution, the equations typically involve solving the Cahn-Hilliard equations [21,22].

Dislocations are essentially two-dimensional (2-D) defects in a continuum three-dimensional (3-D) materials and are typically investigated by electron microscopy. The early Dislocation Dynamics (DD) methods were 2-D [23-25] but recent 3-D Discrete Dislocation Dynamics (DDD) computational approaches [26,27] allow for dislocation motion and interactions with other defects, particles and surfaces.

Most continuum computational methods involve solving relevant Partial Differential Equations (PDE) in which a dependent variable, such as density, is a function of independent variables, usually time and spatial position $[28,29]$. The PDEs are solved together with equations reflecting conservation of mass, energy, and momentum. The computational space is discretized using a 3-D mesh that is either fixed (Eulerian framework) or moves with the computational volume (Lagrangian framework). In the Finite Difference Method (FDM) the solution is obtained using a discrete representation of the PDE. When more flexibility is necessary for complicated geometries, the Finite Element Method (FEM) can provide higherorder approximations and more accurate solutions [30]. Rather than using point approximations on a grid, the Finite Volume Method (FVM) approximates the average integral value of the desired property on a reference volume. Although continuum level methods capture properties of the bulk materials, they are often "informed", by atomistic and meso-scale results.

Several theoretical and computational methods can be grouped under Thermo-Chemical Methods (TCM) [31-33]. They are based on Statistical Mechanics, Solid State Physics, and Thermodynamics and describe chemical properties of the fuel from point defect clusters, to species diffusivity, all the way to the free energy and the kinetics of chemical reactions. Experimental and atomistic results are often the input data for the TCM and the predictive character is tested at continuum level, through experimental characterization of the fuel and the fuel-clad interaction.

For studies of phase stability, the Calculation of Phase Diagrams (CALPHAD) method provides an efficient way to assess the Gibbs free energy models that are consistent with a given set of phase diagram data [34-36]. For example, the CALPHAD method and the temperature integration of the free energy have been coupled to produce phase boundaries in binary systems [37]. In recently published methodologies, part of the free energy models are retrieved from QM calculations [38].

To retrieve known materials properties and validate the calculations it is often necessary to use corrective parameters. In these cases, referring to theoretical and computational method as "first principles" is sometimes an abuse of scientific language. The use of "first principles" should be reserved for calculations that do not involve any fitting to experimentally derived parameters.

\subsection{Models of Nuclear Fuels Properties}

A key component is the understanding the relationships between thermo-mechanical properties such as enthalpy, heat capacity, thermal conductivity, and thermal expansion, and parameters such as temperature, composition, porosity, pressure, and irradiation level. For example, thermal conductivity models as function of temperature have been reviewed [39] but models that include composition as a parameter are quite rare. Several properties are implicitly dependent on the burnup level $[1,2]$. However, correlating thermo-mechanical properties to burnup is a challenging task. A certain burnup level can be associated with a variety of thermo-mechanical properties of the fuel, depending on the history of the fuel element and the damage mechanisms. In other words: predicting fuel properties as function of burnup is highly-desirable; using experimental information that involves burnup as the characteristic parameter may lead to mathematically ill-posed inverse problems.

Understanding of the fuel microstructure (grain size, porosity, and chemistry) and the behavior under irradiation and temperature are also important areas of research. For example, the influence of self and external radiation on void and fission gas bubble formation, swelling, and creep can be incorporated in the thermo-mechanical models [1]. Unfortunately, modeling creep turns out to be very difficult and semi-empirical approaches are currently the only solution.

Fuel materials are not perfect crystals. In the models for properties defective structures, many fuel performance codes are using values from databases that have been measured or calculated for perfect crystals. It is important that to develop new models that incorporate point, line, 2-D, and 3-D defects [40]. Models of dislocations and fracture can play a central role in improving the Finite Element simulations of heat and species transport in fuel elements.

The phase stability of nuclear fuels, especially during transient regimes, is a subject of concern for nuclear energy industry and regulators. Uncertainty evaluations of rather simple phase diagrams, such as $\mathrm{UO}_{2}-\mathrm{PuO}_{2}$ revealed that the solidus and liquidus lines are known within errors as large as $100 \mathrm{~K}$ [41]. Ceramic fuels tend to be less likely to melt but may experience local phase transformations. Metallic fuels are more at risk due the presence of low temperature eutectics in their phase diagram [42]. Since temperature and pressure are the control parameters in most processes, the Gibbs free energy of all phases is the critical thermodynamic property for phase stability calculations [43]. When taking into 
account the fission products and the chemical dynamics of the fuel material, it becomes necessary to study systems with 5-10 components to achieve a good description of the thermo-chemical properties of the fuel. Although free energy functions are available for many phases of interest (mostly oxides and alloys) more work is necessary to cover the nitrides and the advanced (minor actinide containing) fuels. In France, the Atomic Energy Commission (CEA) has developed one of the most advanced databases for thermodynamic properties of fuels, named FUELBASE.

Nuclear fuels are subject to severe radiation environments and their thermal, chemical and mechanical properties change significantly with time and irradiation

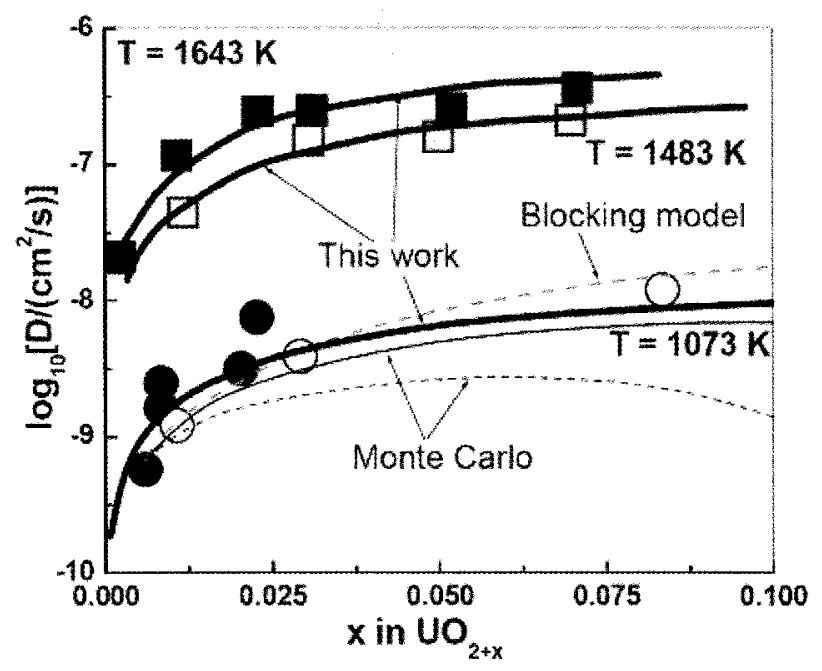

Fig 3. Oxygen Self-Diffusivity as Function of Non-Stoichiometry ( $\mathrm{x}$ in $\mathrm{UO} 2+\mathrm{x}$ ) and Temperature. Symbols: Data from Literature. The Model [54] is Valid for a Large Domain of Temperature $(300 \mathrm{~K}<\mathrm{T}<1800 \mathrm{~K})$ and Oxygen Content $(\mathrm{x}<0.1)$
Fig 2. Cross-Section of UO2 Fuel Rod Operated at a Linear Power High enough to cause Extensive Melting [44]
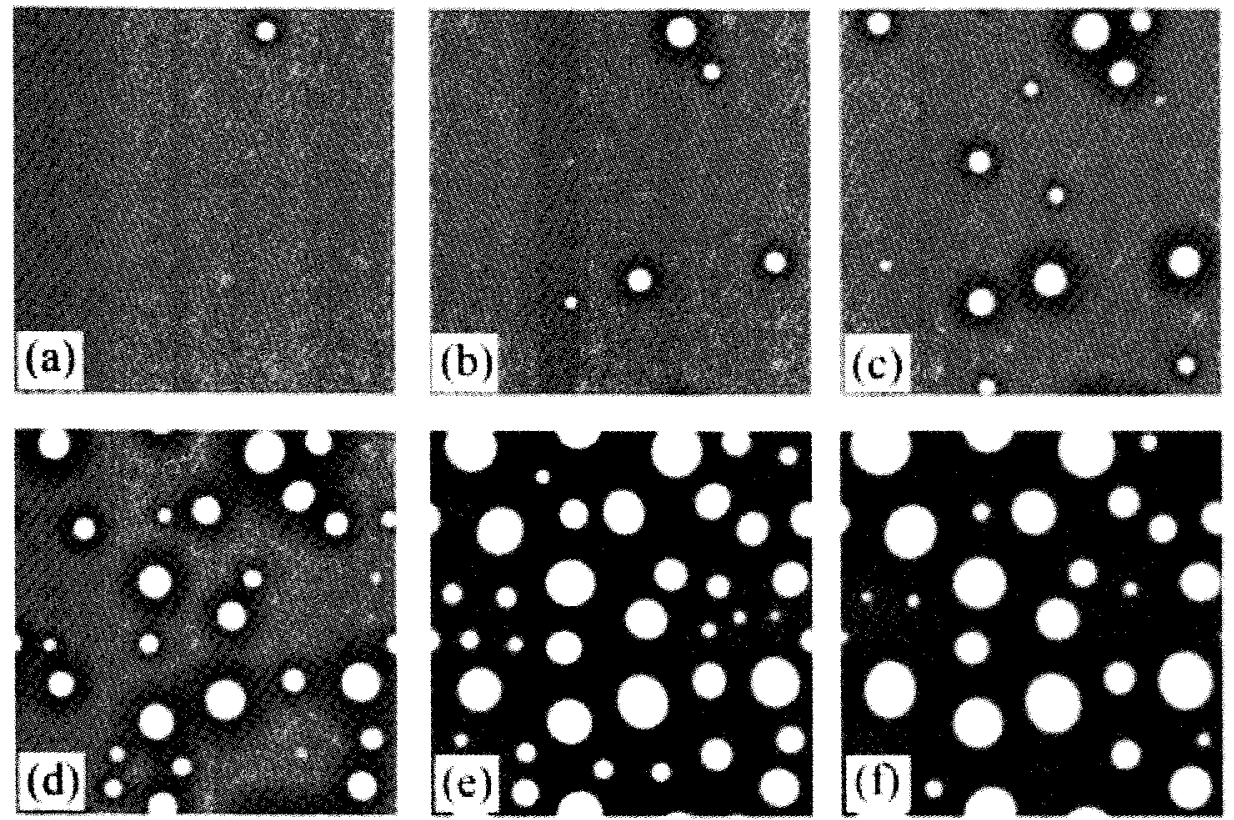

Fig.4. Phase Field Simulation of Homogeneous Nucleation and Growth of Gas Bubbles. Characteristic Time: (a) 10s, (b) 50s, (c) 100s, (d) $150 \mathrm{~s}$, (e) $250 \mathrm{~s}$, (f) $350 \mathrm{~s}$. Next Step is Including Microstructure Features, Especially Grain Boundaries [47] 
level [1]. The major factors that influence the properties are temperature, stoichiometry, and microstructure (especially porosity and point defects). It was discovered early on [44] that the accumulation of fission products in gas bubbles can decrease the heat transfer, leading to overheating of the fuel element and local melting (Fig. 2).

Recent work at Los Alamos National Laboratory (LANL) was focused on irradiation effects on properties such as thermal conductivity, oxygen diffusivity, and thermal expansion. The methods cover a large spectrum of time and space scales, from electronic structure to atomistic, to meso-scale, to continuum [45]. As an example of the coupling between atomistic models of point defect and thermochemical methods, Fig. 3 shows a calculation of oxygen diffusivity in $\mathrm{UO}_{2+\mathrm{x}}$. The model is further used to predict point defect concentrations and fuel stoichiometry at various temperatures and oxygen pressures. In these results, the migration rates of the oxygen interstitials and vacancies are calculated using activation energies obtained from experiments [46].

Another example, this time at the meso-scale, is given in Fig. 4 and shows the gas bubbles formation and evolution [47].

Current work in this area in USA, France, and Japan is focused on developing models of advanced, multicomponent fuels that contain transuranic elements (minor actinides). The next step is to include fission products as system components.

\subsection{Simulations of Phenomena in the Nuclear Fuel Element}

Uranium oxide based nuclear fuels are commonly used in thermal, light water reactors and have been recently considered as the potential fuel for fast, breeder reactors. As shown in Fig. 5, the oxide nuclear fuel rods consist of oxide fuel pellets stacked in a cylindrical metal cladding and then bundled in a fuel assembly, operating

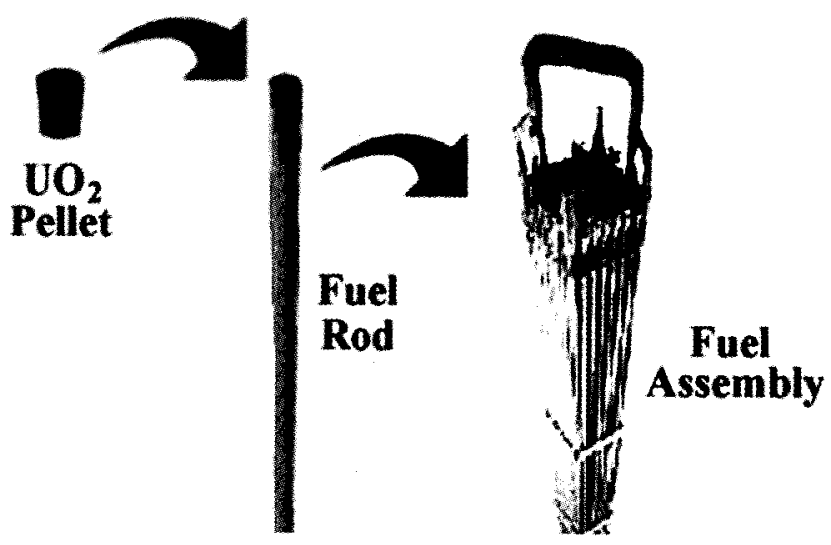

Fig. 5. Schematic of the Nuclear Fuel Rod and Assembly [48] at temperatures up to $2000 \mathrm{~K}$ [48].

There are many fuel types and evaluating them is beyond the scope of this work. The most popular, with the richest history, are based on uranium oxide [49]. Plutonium oxide was introduced as a component of the Mixed Oxide Fuels (MOX) for reasons that include reprocessing of the weapons grade plutonium metal for energy purposes.

Given the tremendous resources (cost, time, people) required to conduct experiments in the nuclear reactors, the studies of nuclear fuels is sometimes replaced by studies on surrogate ceramic materials, such as ceria $\left(\mathrm{CeO}_{2}\right)$ or zirconia $\left(\mathrm{ZrO}_{2}\right)$. The problem with such studies is the poor transferability of the results. For example, although ceria is considered a good surrogate for plutonia [50,51] it is not clear if the study of the fission product diffusion mechanism in the $\mathrm{UO}_{2+\mathrm{x}}-\mathrm{CeO}_{2-\mathrm{x}}$ system can provide a definitive answer to the questions related to fission products accumulation in a mixed oxide fuel $\mathrm{UO}_{2+\mathrm{x}}-\mathrm{PuO}_{2-\mathrm{x}}$.

Researchers at LANL have examined the influence of temperature and stoichiometry changes on the $\mathrm{UO}_{2+\mathrm{x}}$ fuel properties and on the coupling of heat and species transport in a fuel element with stainless steel cladding [52]. The objective was to improve the understanding of fuel damage and performance.

In the nuclear reactor irradiation induces changes in material properties such as microstrocture, density, thermal conductivity, specific heat, and oxygen diffusivity. Several models have been developed for these properties using as parameters the temperature, pressure, burnup, and other reactor parameters [53-54]. As opposite to common approaches the LANL models include the dependence of the properties on the stoichiometry $x$ in $\mathrm{UO}_{2+\mathrm{x}}$.

The finite element simulations of coupled heat and oxygen transport were performed using COMSOL Multiphysics which provides an ideal tool for studying coupled phenomena and allows for mesh and time step refinement in 3-D configurations (Fig. 6). Quadratic Lagrange elements and a non-linear iterative technique with a nested un-symmetric multi-frontal (UMFPACK) linear solver have been used to solve the coupled heat and species equations.

The steady-state parametric studies were focused on

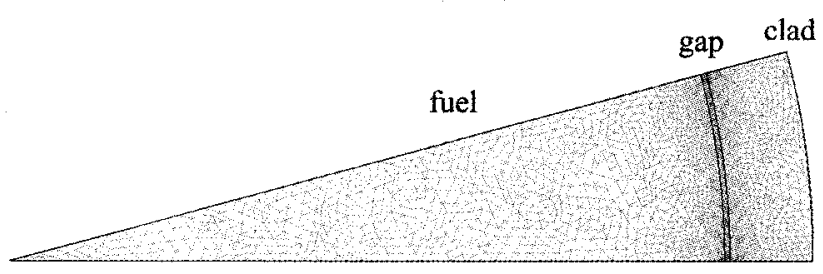

Fig. 6. Representative "Slice" of the Computational Domain, Showing a 22.5 Deg. Angular Sector of the Fuel Element. The Mesh was Refined at the Gap, where Temperature and Stoichiometry Gradients are Steeper 


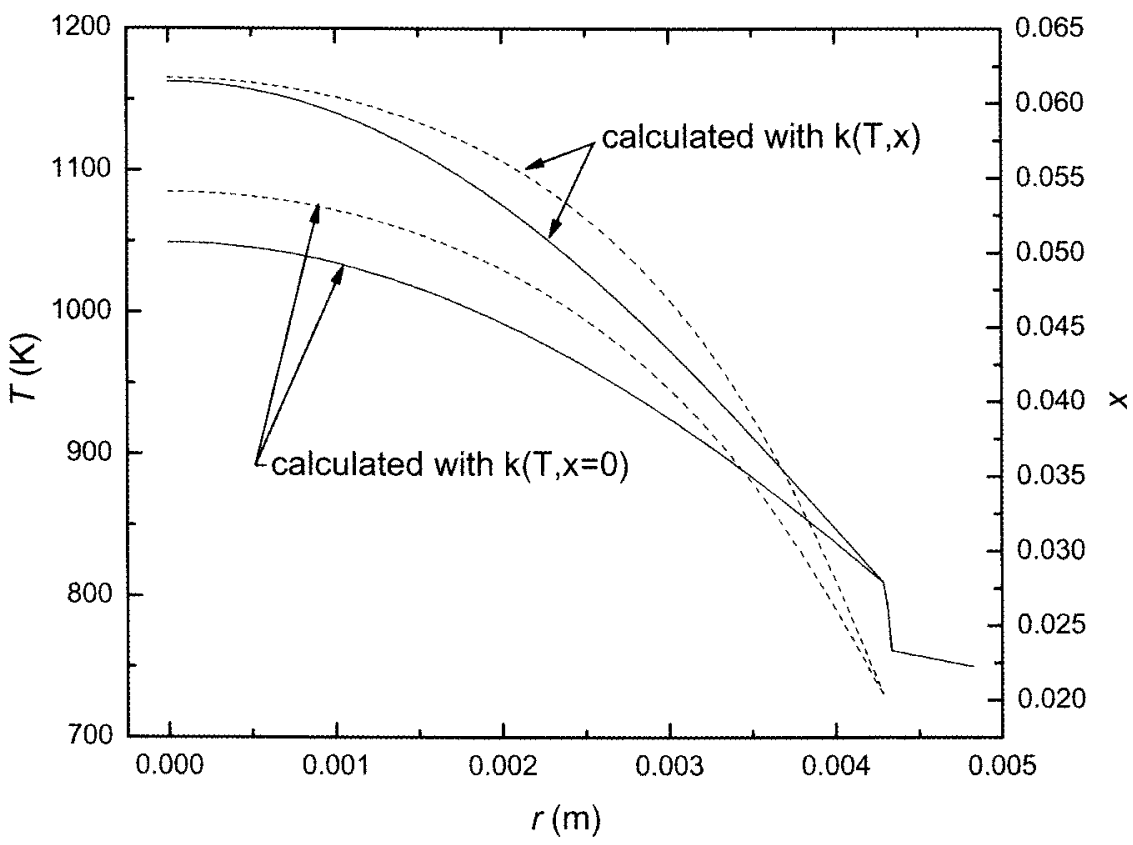

Fig. 7. Steady State Temperature (Solid Lines) and Non-Stoichiometry (Dashed Lines) Profiles Calculated with and without $\mathrm{x}$ Dependence on the Expression for Thermal Conductivity of the $\mathrm{UO}_{2+x}$ Fuel. Note the Significant Changes Introduced by Including the Stoichiometry Dependence of the Thermal Conductivity in the Simulation Compared to Temperature Dependence Only

determining the centerline temperature in the fuel rod as a function of non-stoichiometry and the rate of heat generation during fission. Given the strong temperature gradients in the reactor, the effect of thermally driven diffusion of species, also known as the Soret effect, had to be included. The results show that the counterbalancing of the Soret $[55,56]$ and Fickian fluxes is responsible for the variation of oxygen concentration in the fuel pellet [52]. The simulations demonstrate (Fig. 7) that including the dependence of thermal conductivity and density on non-stoichiometry can lead to changes in the calculated centerline temperature and thermal expansion displacements that exceed $5 \%$.

Additional simulations involved transient regimes and examination of the time lag in the response of the temperature and non-stoichiometry distributions with respect to sudden changes in heat generation rate intensity and oxygen removal rate. Current work includes studies of the effects of porosity and simulations of fuel-cladding interactions.

\subsection{Fuel Performance Codes}

Evaluating the properties of the fuel and predicting the change in properties caused by the reactor environment is a challenging task. Most nuclear technological processes are complex combinations of the reviewed properties and phenomena. As an example, sintering of ceramic fuel materials involves heat and mass transport, phase transformations, irradiation effects, and changes in mechanical properties. A complex simulation of the sintering process must be developed as soon as possible, to assist with fuel design and fabrication. Fuel performance is one of the areas that already benefited from models and simulations. In addition to extensive experimental Post Irradiation Examination (PIE), the fuel performance codes are increasingly used in support of fuel characterization and optimization.

A fuel performance capability (FPC) consists of a computer code or a set of codes that include models of fuel properties and are able to simulate phenomena in the nuclear fuel during operation. In a more general definition of this concept, the fuel performance is evaluated in the fuel element (fuel plus clad) and the applicability is extended to cover manufacturing and storage.

The FPCs are sometimes classified according to their history and complexity into "generations" starting with Generation 1 (1-D, serial codes based on empirical models) and ending with Generation 4 (future, parallel, 3-D codes that contain theory based models).

A review of commercial fuel performance codes show that most countries tend to develop their own simulation capabilities. Here is a list (by no means exhaustive) of some of the most popular fuel performance codes: COMETHE (Belgonucleaire, Belgium), COPERNIC (FRAMATOME, Germany), ENIGMA (British Energy, BNFL, UK), FALCON (EPRI, USA), FRAPCON (PNNL, USA), FRAPTRAN (PNNL, USA), LIFE (ANL, USA), 
MACROS (SCK-CEN, Belgium), ORIGEN (ORNL, USA), PARFUME (INEEL, USA), SPHERE (PSI, Switzerland), TRANSURANUS (ITU, Germany).

The major drawback of most commercial codes is the fact that they are too dedicated to specific fuel types and compositions. The use of empirical correlations in describing materials properties and the much simplified description of heat and mass transport phenomena make extrapolations and information transfer impossible. In order to achieve a consistent predictive character, many codes are moving away from empirical models and include theory based models.

No doubt that neutron transport fission processes, although not the main subject of this work, are a critical components of fuel behavior. Phenomena such as fission products release, diffusion, and accumulation have a strong impact on materials properties and influence the heat and mass transport. Up to now, nuclear reactions have been decoupled from the study of material properties. For example, in many commercial fuel performance codes, the heat generated during fission is only added as a source term to the finite element calculations.

Since in the nuclear fuels community heat and species transport phenomena have been studied in much more detail, most commercial fuel performance codes, such as FRAPCON [57] have capabilities can globally evaluate the results of heat transfer and species diffusion but cannot actually simulate the phenomenon. Some codes can address transient regimes and solve for the time dependent transport equations. More challenging are the simulations of heat transfer in heterogeneous materials, with porosity and defects distributed in the fuel rod.

Although extremely important, the diffusion of fission products is less understood due to the lack of "in situ" characterization methods. Also, the role of diffusion at the grain boundaries is still unclear. This phenomenon is strongly related to microstructure evolution and must be further studied using experimental and theoretical tools. Unfortunately, accurate models of microstructure evolution (point defects, dislocations, and with grain boundary movement) during service are most often lacking absent from commercial fuel performance codes.

The review shows that many codes do not address the thermo-chemistry of chemical reactions, such as oxidation/ reduction or the effect of the coolant on the radiationenhanced corrosion of reactor materials. One exception is TRANSURANUS, which accounts for radial redistribution of oxygen in fast breeder reactor fuels.

Most commercial fuel performance codes focus on thermo-mechanical properties. Still, mechanical phenomena that involve 3-D simulations, such as pellet fragmentation and clad-pellet interactions are difficult to address at this

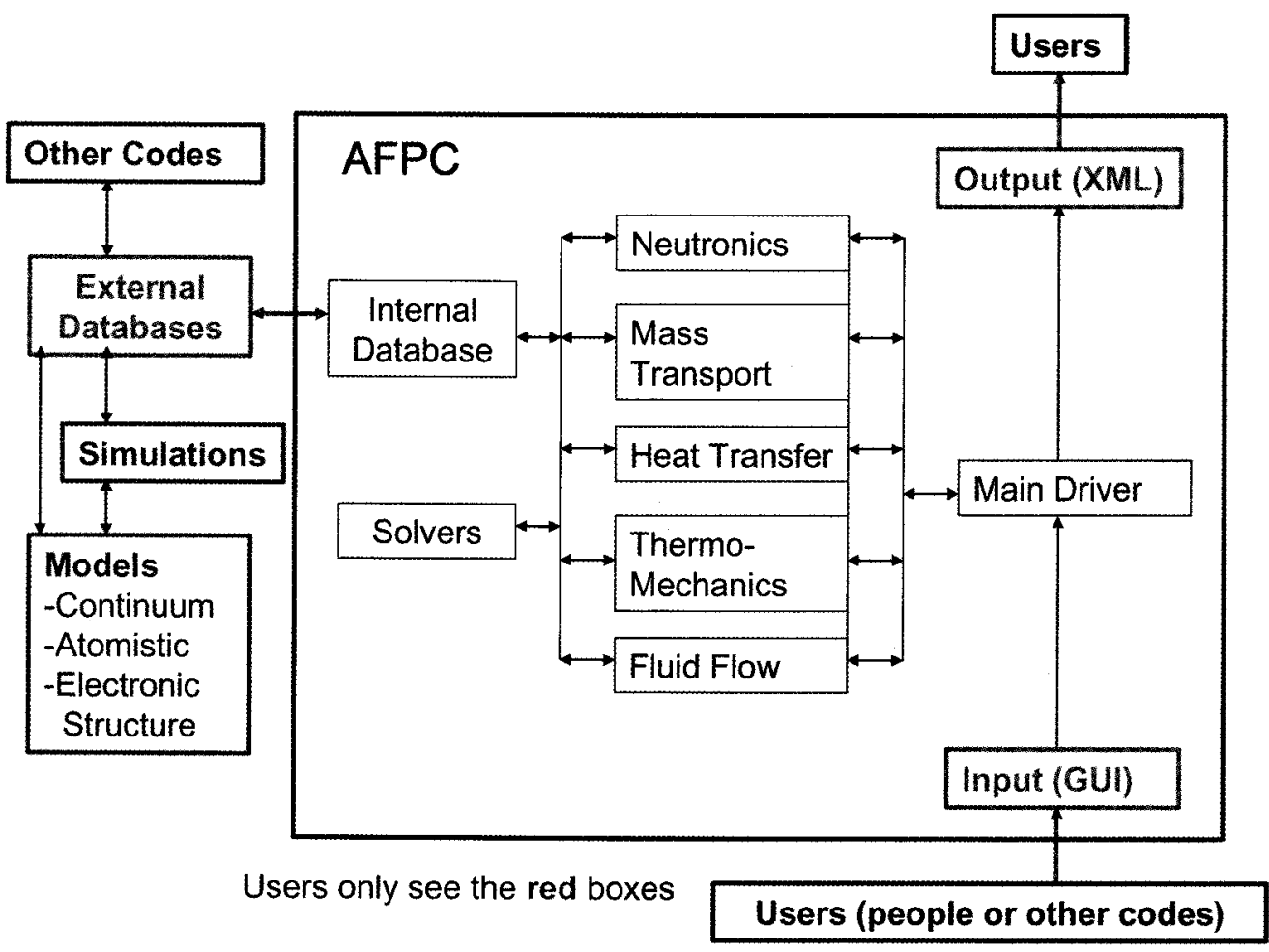

Fig. 8. Preliminary Design of the Advanced Fuel Performance Code (AFPC) [58] 
stage. Most commercial codes do not allow for simulations of large changes in the gap and clad geometry. That leads to simulation tools for rather idealized geometries, difficult to validate against experimental results on real fuel elements.

From a computational point of view, a major problem is the fact that commercial codes cannot run parallel on high-performance computational networks. That increases the computation time and infringes upon the use of complex models. Today's supercomputers are faster and faster. Each of the top 500 fastest supercomputers in the world can now reach over a teraflop per second. At the time of writing this article, the number one position in the world was again claimed by the Roadrunner computer at LANL, a joint development of IBM, Toshiba, and the U. S. A. National Nuclear Security Administration (NNSA). The second place, although the faster computer for open (unclassified applications), was held by the Jaguar computer at Oak Ridge National Laboratory, also in U. S. A. The European Union and Japan are working on systems that can definitely compete for the first place. It is unfortunate that current fuel performance codes do not take advantage of this technology and are still running on personal less performant platforms. There is a tremendous opportunity for moving to high performance computing. Already, neutron diffusion calculations, as well as some safety and security simulations, are performed on such resources. The fuel performance codes can and must become more complex to run on supercomputing networks.

Another major difficulty is the lack of compatibility between the commercial fuel performance codes. The codes have very useful complementary features but cannot be coupled and executed together. It is also difficult to have them feed information to each other. Software engineering issues prevent a full coupling of the codes and make very difficult modifying them to run in parallel, as required for large scale simulations on advanced computational platforms. To solve this problem, France has developed the integrating platform SALOME, intended to facilitate the creation of industrial simulation applications. It is currently used by CEA for nuclear energy simulations that incorporate independent fuel performance and reactor codes.

It is known that there is much experience accumulated in developing commercial fuel performance codes is due to the work of materials scientists and nuclear engineers with exceptional computational skills. The codes include good descriptions of the fuel properties and associated phenomena but the software engineering side is modest. Another class of software consists in programs developed for complex simulations for national security applications. These are much more powerful computational tools but they lack the necessary models to describe nuclear fuels.

The question is: Is it better to attempt to modify existing coeds to make them structured, run in parallel on high performance computational platforms, and possibly become object oriented, OR create new codes from scratch? There are many advantages in improving upon the available commercial codes, the main being that most of them have been validated and work well within their scope. However, there are several options for developing new fuel performance capabilities, going from integration of existing codes to the design of new ones.

Developing a new fuel performance code is a complex, challenging endeavor. Figure 8 shows a preliminary design of an Advanced Fuel Performance Code (AFPC) [58]. To address the nuclear fuels material properties and phenomena, the code must include, at the minimum, the following modules:

- Neutronics (fission and neutrons diffusion)

- Heat transfer (conduction, convection, and radiation)

- Mass transport (species diffusion and gas accumulation)

- Thermo-mechanics (deformation, such as swelling)

- Fluid flow (to model the coolant, if necessary)

There are also requirements related to numerics and code design, such as using nested (linear + non-linear) solvers, multi-level preconditioning, running parallel via MPI on multi-CPU platforms, and easy adaptation to hybrid platforms. The main driver of the code must interact with an external database that is continuously updated with the most advanced models of fuel and materials properties and the necessary nuclear data. Given the improvements in precision and accuracy, and the full validation of the methods, the computer simulations capabilities (sets of codes) are likely to soon become part of the fuel qualification process.

\section{INTERNATIONAL COLLABORATIONS}

Besides better experiments, models, simulations and computational capabilities, a coherent nuclear energy program requires national and international collaborations. Given the tremendous amount of work and the highly innovative character of the research, scientists often team up in developing new theories and complex software packages. To cover the necessary area of expertise, organizing workshops and sessions on models and simulations of nuclear materials at international meetings appears to be the natural solution.

\subsection{The Materials Models and Simulations for Nuclear Fuels (MMSNF) Workshop Series}

The Materials Models and Simulations for Nuclear Fuels (MMSNF) workshop series aims at stimulating discussions and research to advance theory-based model development, high-performance computer simulations, and experimental validation for nuclear fuels applications.

The workshops series started in June 9-10, 2003 in Santa Fe, NM, USA [59], with support from the Advanced Fuel Cycle Initiative (AFCI) program, funded by the USA Department of Energy and LANL. It brought together fifty experts in models and simulations from 
United States, UK, Sweden, and Turkey. This first edition was focused on Materials Models and Simulations, with less emphasis on fuel performance codes. Presentations detailed results of electronic structure, atomistic, and continuum simulations. The workshop ended with a plenary discussion of the role of modeling and simulations in designing better nuclear fuels.

The second edition, November 20-21, 2003, was organized in New Orleans, LA, USA [60]. Forty participants attended the meeting, with Belgium, Germany, Japan, and the Netherlands as new members of the community. On the USA side, six National Laboratories were represented. University participation decreased compared to the first edition, but there was a slight increase in attendance from industry/private companies.

The third edition of the workshop shifted the focus of the discussions from materials science to fuel performance predictive codes, design, manufacturing, and operation [60]. Organized on November 18-19, 2004, in Washington DC, USA, the meeting was scheduled in connection with the winter meeting of the American Nuclear Society. Canada, France and Japan were the new members and the USA national laboratories, universities, and private companies were more evenly represented. After three editions, the number of scientists, engineers, and managers involved in the MMSNF workshops reached a relatively stable number of 50 . The participants included materials scientists, physicists, chemists, nuclear engineers, computational scientists, software engineers, and managers.

After a fourth edition, still in Washington DC, November 17-18, 2005, the workshop went international to provide a better venue for participants on all continents [61]. The fifth edition was organized in Nice, France, on June 1-2, 2006 with support from the Institute de Radioprotection and Nuclear Safety (IRSN, France) [62]. The edition was successful in many respects, including participation of national research institutions such as CEA, the Japan Atomic Energy Agency (JAEA), the Institute for Transuranic Elements (ITU, European Community). Nuclear regulatory organizations have been also represented. Besides IRSN, the participants included The Nuclear Regulatory Commission (NRC, USA), the Nuclear Safety institute (IBRAE, Russia), and the Italian Nuclear Energy Agency (ENEA).

Organized in Tokyo, MMSNF-6, December 13-14, 2007 benefited from support of the University of Tokyo and re-focused on the multi-scale aspects of model development [63]. A strong presence from Europe, Japan, and the Republic of Korea ensured the success of the workshop.

The seventh edition of the workshop was organized in Karlsruhe, Germany, September 22-23, 2008, by the European Commission and ITU [64]. The presentations included state of the art models and simulations covering ES, MD, PF, and FEM methods and ended with a session dedicated to fuel performance codes. At the time of the publication of this article, MMSNF-8 is in preparation, with a proposed date in October 2009, in Albuquerque, NM, USA.

During the workshops and follow up discussions, various models and simulation methods have been analyzed and participants made suggestions regarding long and short-term theoretical efforts that can help with improving the models. One of the important observations was that the work on nuclear (ceramics) fuels is way behind the work on metals and must catch up as soon as possible. There is a tremendous opportunity for moving nuclear fuels simulations towards high performance computing.

Several workshop participants supported the idea of creating national and international Centers for Models and Simulations of Nuclear Fuels. This is a more general concept than models and simulations of materials for nuclear fuels. It involves all components of nuclear fuels design and optimization: materials, neutron fluxes, test beds, controls, non-proliferation, safety and security. The mission of such centers would be to provide the scientific environment and resources (people, supercomputers, and funding) for the development of theory-based models, simulations, and computational tools for the design and optimization of nuclear fuels.

Following the third workshop, the International Focus Group (IFG) for Materials Models and Simulations for Nuclear Fuels held its first meeting. The IFG brings together experts in various areas of modeling, simulations, experimental and technological applications from 15 countries. With every edition, the workshops improved the strategy for short and long-term coordination of projects among the participating research groups. In a direct or indirect way, MMSNF workshop series is continuously impacting national strategies in nuclear energy area. It is also a determinant factor in building an international community of scientific experts and decision factors driven by the common interest in creating advanced, innovative nuclear fuels.

\subsection{The Working Party on Multi-Scale Modelling of Fuels and Structural Materials for Nuclear Systems (WPMM)}

Over time, the number of scientists, engineers, and managers attending the MMSNF workshops reached a relatively stable number of 50 . There is however a much larger community interested in the workshop subjects. There is also a need for an organizational framework that nurtures and facilitates interactions among scientists and can assist with scientific publications.

The Organization for Economic Cooperation and Development (OECD) is one of the world's largest and most reliable sources of comparable statistics, and economic and social data [65]. OECD provides a setting where governments compare policy experiences, seek answers to common problems, identify good practice and coordinate domestic and international policies. There are 
26 country members (Australia, Austria, Belgium, Canada, Czech Republic, Danmark, Finland, France, Germany, Greece, Hungary, Iceland, Ireland, Italy, Japan, Korea, Luxembourg, Mexico, the Netherlands, New Zeeland, Norway, Poland, Portugal, Slovak Republic, Spain, Sweden, Switzerland, Turkey, United Kingdom, and United States). In May 2007, OECD countries agreed to invite Chile, Estonia, Israel, Russia and Slovenia to open discussions for membership and offered enhanced engagement, with a view to possible membership, to Brazil, China, India, Indonesia and South Africa.

One of the scientific components of OECD is the Nuclear Energy Agency (NEA). The goal of the NEA is to help member countries identify, develop, and disseminate basic scientific and technical knowledge required to ensure safe, reliable and economic operation of current nuclear systems and to develop new technologies [66]. The NEA nuclear science program is developed by the Nuclear Science Committee (NSC), comprising high-level scientific experts from all NEA member countries, and executed mainly by subsidiary Working Parties (WP) and Expert Groups (EG).

The Working Party on Multi-scale Modelling of Fuels and Structural Materials for Nuclear Systems (WPMM) was created in January 2008 to address scientific and engineering aspects of fuels and structural materials. The main goal is to establish multi-scale models and simulations as validated predictive tools for the design of nuclear systems, fuel fabrication and performance. The main tasks include: identification of fundamental problems, development of FP and atomistically-informed models and simulations of nuclear fuels and structural materials properties, promoting high performance computer simulations, and creating and maintaining synergy with experimental work. Validation of simulations and model predictions by benchmarking is also a priority, together with creating and maintaining the synergy with experimental work. And the development of new applied mathematics and software tools is strongly encouraged.

At the second WPMM meeting, Sept, 2008, Paris, France, five Expert Groups have been defined: A) Nuclear Fuels B) Structural Materials C) Multi-Scale Methods D) Validation Experiments and E) Uncertainty Evaluation. The third meeting will be in Paris, March, 30-31, 2009. At the time of writing this article, WPMM was accepting nominations for the expert groups.

\subsection{Human Resources}

It is well accepted that improved experimental and computational capabilities are necessary to create better nuclear fuels. However, the most important resources are the people, the nuclear engineers. Their contribution is essential in ensuring the relevance of the modeling and simulation work and they are the supreme validation authorities. Scientists are often tempted to direct their attention toward the most interesting and challenging areas, rather than the most relevant ones. MMSNF and WPMM meetings proved that an increasing number of nuclear engineers are familiar with complex simulation tools and are currently using them for optimizing fuel properties. They are the customers of the materials models and simulations work and will benefit the most from the development of theory-based fuel performance codes.

As previously pointed out, for a long time modeling and simulation work was dedicated to alloys and there is a wealth of methods developed for metallic systems. With a few exceptions, the computational materials science community is less interested in ceramics, not to mention actinide based ceramics. The recent involvement of several prestigious physicists, chemists, and materials scientists in developing models of ceramic nuclear fuels properties was viewed as a successful start. Their involvement was driven by the interest in solving challenging scientific problems associated with nuclear fuels development, and to a lesser degree by funding.

No doubt computational scientists and software engineers are the key participants in designing and writing the fuel performance code. The question is: should they have the lead in this process? Strong teaming among engineers, scientist, and software developers appears to be the solution. Hopefully, the increasing international collaborations will provide a better justified answer.

The involvement of the decision factors at national and international levels in the meetings and their active participation in discussions is one of the most valuable developments. Not only that the decision factors became aware of the scientific and computational challenges faced by the models and simulations community, but they provided guidance and support for future programs.

To expand and improve the quality of the models and simulations, the international nuclear fuels community needs to develop a large pool of experts to cover all necessary theoretical, experimental, and computational tasks. That can be achieved by including "Models and Simulation" in the materials science and nuclear engineering programs at universities all over the world.

\section{CONCLUSIONS}

The physics and chemistry of nuclear fuels are complex due to irradiation effects and there is a need for a theory-based approach to develop a fundamental understanding of properties of complex nuclear fuel materials in the reactor environment. A key component is relating thermo-mechanical properties such as enthalpy, heat capacity, thermal conductivity, and thermal expansion, to parameters such as temperature, composition, porosity, pressure, and irradiation level. This will lead to improved tools for predicting reactor phenomena, including heat transfer, phase stability, species diffusion, and fission 
products retention.

Models and simulations address a wide range of space and time scales, starting with the nucleus, to the electronic structure, atomistic and nano-scale, mesoscale, all the way to the fuel element size (centimeters), and from pico-seconds, to seconds, all the way to the operating and storage characteristic times (months, years). There is a variety of multi-scale methods available for nuclear fuels. Atomistic methods account for the evolution of each atom in the computation domain, while mesoscale methods operate in time and space intervals that are characteristic to material's nano or micro-structure. Although continuum level methods capture properties of the bulk materials, they are often "informed" by atomistic and meso-scale results.

Recent work at LANL was focused on irradiation effects on properties such as thermal conductivity, oxygen diffusivity, and thermal expansion. By coupling atomistic models of point defect and thermochemical methods, a model of oxygen diffusivity in $\mathrm{UO}_{2+\times}$ was developed. The model was further used to predict point defect concentrations, and fuel stoichiometry at various temperatures and oxygen pressures. The simulations of coupled heat transfer and species diffusion demonstrated that including the dependence of thermal conductivity and density on composition can lead to changes in the calculated centerline temperature and thermal expansion displacements that exceed $5 \%$.

Advanced nuclear fuel performance codes should incorporate theory-based models and simulations rather than empirical correlations. The major drawback of most commercial codes is the fact that they are too dedicated to a specific fuel forms and compositions. The use of empirical correlations in describing materials properties and the much simplified description of heat and mass transport phenomena make extrapolations and information transfer difficult. In order to achieve a consistent predictive character, many codes are moving away from empirical models and include theory based models. From a computational point of view, a major problem is that the majority of commercial codes cannot run parallel on high-performance computational networks.

Besides better experiments, models, simulations and computational capabilities, a coherent nuclear energy program requires national and international collaborations. The Materials Models and Simulations for Nuclear Fuels (MMSNF) workshop series aims at stimulating discussions and research to advance theory-based model development, high-performance computer simulations, and experimental validation for nuclear fuels applications. The main goal of the Working Party on Multi-scale Modelling of Fuels and Structural Materials for Nuclear Systems (WPMM) is to establish multi-scale models and simulations as validated predictive tools for the design of nuclear systems, fuel fabrication and performance. However, the most important resources is the people: scientists, engineers, and managers. Their contribution is essential in ensuring the relevance of the modeling and simulation work. To expand and improve the quality of the models and simulations, the international nuclear fuels community needs to develop a large pool of experts to cover all necessary theoretical, experimental, and computational tasks. That can be achieved by including "Models and Simulation" in the materials science and nuclear engineering programs at universities all over the world.

In addition to extensive experimental Post Irradiation Examination (PIE), the fuel performance codes are increasingly complex tools in support of fuel characterization and optimization. Given the improvements in precision and accuracy and the full validation of the models, the computer simulations capabilities are likely to soon become part of the fuel qualification process.

\section{REFERENCES}

[ 1 ] D. R. Olander, "Fundamental Aspects of Nuclear Reactor Fuel Elements", TID-26711-P1, Technical Information Service, U.S. Department of Commerce, Springfield, Virginia (1976).

[2] W. M. Stacey, Nuclear Reactor Physics, John Wiley \& Sons, Inc, New York (2001).

[3] A. P. Sutton, Electronic Structure of Materials, Oxford University Press, Oxford (1993).

[4] J. Singleton, Band Theory and Electronic Properties of Solids, Oxford University Press, Oxford (2001).

[ 5 ] R. M. Martin, Electronic Structure, Cambridge Univ. Press, New York (2004)

[6] N.H. March, Electron Density Theory of Atoms and Molecules, Academic Press, New York (1992).

[7] W. Kohn and L. J. Sham, "Self-Consistent Equations Including Exchange and Correlation Effects", Phys. Rev. $A, \mathbf{1 4 0}, 1133$ (1965).

[8] K. Kunc and R. M. Martin, "Ab Initio Force Constants of GaAs: A New Approach to Calculation of Phonons and Dielectric Properties", Phys. Rev. Lett., 48, 406 (1982).

[9] W. Frank and C. Elsasser and M. Fahnle, "Ab initio ForceConstant Method for Phonon Dispersions in Alkali Metals", Phys. Rev. Lett., 74, 1791 (1995).

[10] D. Frenkel and B. Smit, Understanding Molecular Dynamics, Academic Press, San Diego (2002).

[11] G. D. Billing and K. V. Mikkelsen, "Introduction to Molecular Dynamics and Chemical Kinetics", John Wiley \& Sons, Inc, New York, 1996.

[12] M. S. Daw and M. I. Baskes, "Embedded-Atom-Method Functions for the fec Metals $\mathrm{Cu}, \mathrm{Ag}, \mathrm{Au}, \mathrm{Ni}, \mathrm{Pd}, \mathrm{Pt}$, and Their Alloys", Phys. Rev. B 29, 6443 (1984).

[13] M. I. Baskes, "Modified Embedded-Atom Potentials for Cubic Materials and Impurities", Phys. Rev. B 46, 2727 (1992).

[14] G. Busker, A. Chroneos, and R. W. Grimes, "Solution Mechanisms for Dopant Oxides in Yttria", J. Am. Ceram. Soc., 82, 1553 (1999).

[15] M. Abramowski, "Atomistic Simulations of the Uranium/ Oxygen System”, PhD Thesis, Imperial College (2001).

[16] A. F. Voter, F. Montalenti, and T. C. Germann, "Extending 
the Time Scale in Atomistic Simulations of Materials", Ann. Rev. Mater. Res., 32, 321 (2002).

[17] N. Metropolis and S. Ulam, "The Monte Carlo Method", J Amer. Statistical Assoc., 44, 335 (1949).

[18] A. F. Voter, "Introduction to the Kinetic Monte Carlo Method", in Radiation Effects in Solids, K. E. Sickafus and E. A. Kotomin (Editors), Springer, NATO Publishing Unit, Dordrecht, The Netherlands (2005).

[19] W. M. C. Foulkes, L. Mitas, R. J. Needs, and G. Rajagopal, "Quantum Monte Carlo Simulations of Solids", Rev. Mod. Phys. 73, 33 (2005).

[20] V.L. Ginzburg and L.D. Landau, "On the theory of superconductivity", Zh. Eksp. Teor. Fiz. 20, 1064 (1950).

[21] J.W. Cahn. "On Spinodal Decomposition", Acta Metall., 9, 795 (1961).

[22] A Karma. "Phase-Field Formulation for Quantitative Modeling of Alloy Solidification", Phys. Rev. Lett., 87, 115701 (2001).

[23] J. Lepinoux and L. P. Kubin, "The Dynamic Organization of Dislocation Structures: A Simulation", Scripta Metall. 21, 833 (1987).

[24] E. Van der Giessen and A. Needleman, A., "Discrete Dislocation Plasticity: A Simple Planar Model", Mater. Sci. Eng. 3, 689 (1995).

[25] H. Y. Wang and R. LeSar, "O(N) Algorithm for Dislocation Dynamics", Philosophical Magazine A, 71, 149 (1995).

[26] J. P. Hirth, M. Rhee, and H. M. Zbib, "On Dislocation Reactions and Hardening Mechanisms in 3D Dislocation Dynamics", J. Computer-Aided Materials Design 3, 164 (1996).

[27] V. Bulatov, M. Tang, M. and H. M. Zbib, "Crystal plasticity from dislocation dynamics", Materials Research Society Bulletin 26, 191 (2001).

[28] F. P. Incopera and D. P. DeWitt, Fundamentals of Heat and Mass Transfer, John Wiley \& Sons, Inc, New York (1996).

[29] R. B. Bird, Transport Phenomena, John Wiley \& Sons, Inc, New York (2002).

[30] J. N. Reddy and D. K. Gartling, The Finite Element Method in Heat Transfer and Fluid Dynamics, CRC Press, LLC, Boca Raton (2001).

[31] A. R. Allnatt and A. B. Liliard, Atomic Transport in Solids, University Press, Cambridge, New York, 1993.

[32] S. I. Sandler, Chemical Engineering and Thermodynamics, John Wiley \& Sons, Inc, New York (1999).

[33] M. E. Glicksman, Diffusion in Solids, John Wiley \& Sons, Inc, New York (2000).

[34] L. Kaufman and H. Bernstein, Computer Calculations of Phase Diagrams, Academic Press, New York (1970).

[35] N. Sounders and A. P. Miodownik, CALPHAD, Elsevier Science Limited, New York (1998).

[36] M. Hillert, Phase Equilibria, Phase Diagrams and Phase Transformations, Cambridge University Press, New York (1998).

[37] M. I. Baskes and M. Stan, "An Atomistic Study of SolidLiquid Interfaces and Phase Equilibrium in Binary Systems", Metall. Mater. Trans. A, 34, 435 (2003).

[38] M. I. Baskes, K. Muralidharan, M. Stan, S. M. Valone, and F. J. Cherne, "Using the Modified Embedded-Atom Method to Calculate the Properties of Pu-Ga Alloys", JOM, 55, 41 (2003).
[39] J. K. Fink, "Thermophysical Properties of Uranium Dioxide", J. Nucl. Mater., 279, 1 (2000).

[40] R. B. Phillips, Crystals, Defects, and Microstructures, Cambridge University Press, Cambridge (2001).

[41] M. Stan and B. Reardon, "A Bayesian Approach to Evaluating the Uncertainty of Thermodynamic Data and Phase Diagrams", CALPHAD, 27, 319 (2003).

[42] S. S. Hecker and M. Stan, "Properties of Plutonium and its Alloys for Use as Fast Reactor Fuels" J. Nucl. Mater. 383, $112(2008)$.

[43] T. B. Lindemer and T. M. Besmann, "Chemical Thermodynamic Representation of UO2 $\pm \mathrm{x}$ ", J. Nucl. Mater., 130, 473 (1985).

[44] M. F. Lyons, B. Weidenba, R.F. Boyle, and T. J. Pashos, "Post-Irradiation Examination of High-Burnup Molten UO2 Fuel Rods", Trans. Amer. Nucl. Soc., 8, 42 (1965).

[45] M. Stan, J. C. Ramirez, P. Cristea, S. Y. Hu, C. Deo, B. P. Uberuaga, S. Srivilliputhur, S. P. Rudin, and J. M. Wills., "Models and simulations of nuclear fuel materials properties", J. Alloys Comp., 444-445, 415 (2007).

[46] K. C. Kim and D. R. Olander, "Oxygen Diffusion in UO2x “, J. Nucl. Mater., 102192 (1981).

[47] S.Y. Hu, M. I. Baskes, M. Stan and C. Tome, "Phase-field Modeling of Micro-void Evolution under Elastic-plastic Deformation", Appl. Phys. Let., 90, 81921 (2007).

[48] United States Nuclear Regulatory Commission, Emergency Preparedness, http://www.nrc.gov/about-nrc/emergpreparedness/images/fuel-pellet-assembly.jpg

[49] J. Belle (Ed), "Uranium Oxide: Properties and Nuclear Applications", Naval Reactors, Division of Reactor Development Report, USAEC, (1961).

[50] D.G. Kolman, Y.S. Park, M. Stan, R.J. Hanrahan Jr., D.P. Butt, "An Assessment of the Validity of Cerium Oxide as a Surrogate for Plutonium Oxide Gallium Removal Studies", LA-UR-99-0491, Los Alamos National Laboratory (1999).

[51] M. Stan, T.J. Armstrong, D.P. Butt, T.C. Wallace Sr., Y.S. Park, C.L. Haertling, T. Hartmann, R.J.Hanrahan Jr, "Stability of the Perovskite Compounds in the Ce-Ga-O and Pu-Ga-O Systems", J. Am. Ceram. Soc., 85, 2811 (2002).

[52] J. C. Ramirez, M. Stan, and P. Cristea, "Simulations of Heat and Oxygen Diffusion in UO2 Nuclear Fuel Rods", J. Nucl. Mater., 359, 174 (2006).

[53] M. Stan and P. Cristea, "Defects and Oxygen Diffusion in PuO2-x", J. Nucl. Mater. 344, 213 (2005).

[54] P. Cristea, M. Stan, and J. C. Ramirez, "Point Defects and Oxygen Diffusion in Fluorite Type Oxides", J. Optoelectr. Adv. Mater, 9, 1750 (2007).

[55] J. Janek and H. Timm, "Thermal Diffusion and Soret Effect in $(\mathrm{U}, \mathrm{Me}) \mathrm{O} 2+\mathrm{x}$ : The Heat of Transport of Oxygen", $J$. Nucl. Mater., 255, 116 (1998).

[56] C. Korte, J. Janek, and H. Timm, "Transport Processes in Temperature Gradients: Thermal Diffusion and Soret Effect in Crystalline Solids", Solid State Ionics, 101/103, 465 (1997).

[57] FRAPCON simulation code, http://www.pnl.gov/frapcon3/

[58] M. Stan, "Materials Models and Simulations in Support of Nuclear Fuels Development", LA-UR-05-5652, Los Alamos National Laboratory (2005).

[59] http://www.lanl.gov/mst/nuclearfuels/.

[60] http://www.lanl.gov/orgs/mst/mmsnf/.

[61 ] http://public.lanl.gov/mastan/MMSNF/ 
[62] http://workshop_mmsnf5.irsn.org/

[63] http://mmsnf-6.nxo.jp/

[64] http://itu.jrc.ec.europa.eu/index.php?id=36\&type $=$
$\&$ iEntryUID $=164 \&$ iEntryPID $=68$

[65] http:/www.oecd.org/

[66] http:/www.nea.fr/ 